\title{
Thiazole orange-peptide conjugates: sensitivity of DNA binding to chemical structure
}

\author{
Jay R. Carreon, Kerry P. Mahon, Jr., Shana O. Kelley* \\ Boston College, Eugene F. Merkert Chemistry Center, Chestnut Hill, MA 02467
}

\section{Materials}

Solvents were purchased from Fisher and reagents were purchased from Aldrich Chemical Co. (Milwaukee, WI) or Acros Organics (Morris Plains, NJ). Amino acids were purchased from Advanced ChemTech (Louiville, KY). Calf thymus DNA (CT DNA) was purchased from Sigma (St. Louis, MO). All solvents and reagents were used without further purification. HPLC grade acetonitrile and Millipore water were used for HPLC analysis. The buffer used in all experiments was $50 \mathrm{mM}$ sodium phosphate, 10 $\mathrm{mM}$ sodium chloride $(\mathrm{pH} 7)$.

\section{Analysis}

Reversed-phase HPLC was performed using a HP 1100 system with a Varian 250 x $4.6 \mathrm{~mm}$ stainless steel column packed with Microsorb-MV 300 C18 (5 $\square \mathrm{M})$. A flow rate of $1.0 \mathrm{~mL} / \mathrm{min}$. was used with an aqueous solution buffered with $50 \mathrm{mM}$ ammonium acetate and a linear gradient from 20 to $100 \%$ acetonitrile over $80 \mathrm{~min}$. ${ }^{1} \mathrm{H}$ NMR spectra were recorded on a Varian 400 and $500 \mathrm{MHz}$ spectrometer. Proton chemical shifts are reported in ppm $(\square)$ relative to the solvent reference relative to tetramethylsilane (TMS) $\left(\mathrm{d}_{6}\right.$-DMSO, $\square$ 2.50; $\mathrm{CD}_{3} \mathrm{OD}, \square$ 3.30). Data are reported as follows: chemical shift (multiplicity [singlet (s), doublet (d), triplet (t), quartet (q) and multiplet (m)], coupling constants $[\mathrm{Hz}]$, integration). Carbon NMR spectra were recorded on a Varian 500 (125 $\mathrm{MHz}$ ) spectrometer with complete proton decoupling. Carbon chemical shifts were reported in ppm ( $\square$ ) relative to TMS with the respective solvent resonance as the internal standard (DMSO, 39.5; $\mathrm{CD}_{3} \mathrm{OD}, 49.2$ ). Mass spectral analysis was performed by the Boston College Mass Spectrometry Facility. Samples were analyzed by accurate mass electrospray mass spectrometry (ES-MS) operating in positive mode on a Micromass LCT mass spectrometer. UV analysis was performed on a Hewlett Packard 8452A Diode Array Spectrophotometer. Steady state fluorescence measurements were performed on a Jobin Yvon Horiba Fluorolog ${ }^{\circledR}-3$. For all steady state measurements the $\square_{\mathrm{ex}}=470 \mathrm{~nm}$ and the $\square_{\mathrm{em}}=490-650 \mathrm{~nm}$. Dissociation constants were measured using a Perkin Elmer Wallac Victor Fluorescence reader fitted with a 450-490 nm excitation filter and a 515 $\mathrm{nm}$ long pass emission filter. 


\section{Hypochromicity measurements}

Absorbance values for $4 \square \mathrm{M}$ solution of TO-conjugates were measured before and after the addition of $45 \square \mathrm{M}$ (bp) CT DNA. Percent hypochromicity was calculated as the percent change in absorbance at $\square_{\max }$.

\section{Quantum yield measurements}

Quantum yields are reported relative to a $50 \mathrm{nM}$ fluorescein standard in $0.1 \mathrm{M}$ $\mathrm{NaOH}(\square=0.93) .{ }^{1} \quad$ Absorbance values at the excitation wavelength $(470 \mathrm{~nm})$ were measured for solutions containing 13.5 $\square \mathrm{M}$ TO-conjugate and $405 \square \mathrm{M} \mathrm{CT}$ DNA bp. The sample was then diluted to a final TO-conjugate concentration of $1.5 \square \mathrm{M}$ and CT DNA concentration of $45 \square \mathrm{M}$ (bp) to ensure that the absorbance of the sample was $<0.06$ for measurement of emission spectra. The integral of the emission spectrum was corrected for variations in absorbance and reported relative to the fluorescein standard.

\section{Measurement of dissociation constants}

Dissociation constants were determined with fluorescence titrations performed in a 384 well plate with a total volume of $40 \square 1$ in each well. The concentration of TOconjugates was kept constant at $50 \mathrm{nM}$ and the concentration of CT DNA was increased until fluorescence signals plateaued. Each sample was run in triplicate and the values of each concentration point were averaged. Scatchard analysis was used to obtain $\mathrm{K}_{\mathrm{d}}$ values. $^{2}$

\section{Diffusional quenching experiments}

Samples containing 1.5 $\square \mathrm{M}$ TO-conjugate and $45 \square \mathrm{M}$ CT DNA bp were titrated with hexaamineruthenium(III) chloride and the fluorescence was measured after each addition. The data was plotted according to the Stern-Volmer equation (Eq. 1) and the slope of the best-fit line was used to determine the value for $\mathrm{k}_{\mathrm{q}}$. Lifetimes are relative to the reported lifetime for thiazole orange and were based on the ratio of the quantum yield of each sample to thiazole orange $(\square=0.11){ }^{3,4}$

$$
\text { Eq. } \left.1 \quad I_{O} / I=k_{q} \square Q\right]+1
$$

\section{Displacement of distamycin A}

The methods used for the displacement assay are analogous to those described by Boger. ${ }^{5,6}$ Fluorescence of a solution containing 2.0 $\square \mathrm{M}$ TO-conjugate and $12 \square \mathrm{M} \mathrm{CT}$ DNA bp was measured. Distamycin A was added to produce a final concentration of 2.0 $\square \mathrm{M}$ and the fluorescence was remeasured once again. Percent decrease in fluorescence was calculated as the percent change in the integral of the emission spectrum upon addition of distamycin $\mathrm{A}$. 


\section{Synthesis of TO derivatives and TO-peptide conjugates}

Methods were adapted from Svanvik et al. ${ }^{7 \mathrm{a}-\mathrm{c}}$

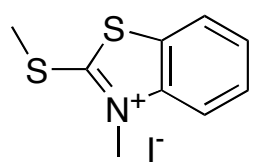

\section{Preparation of compound 1}

To a dry $50 \mathrm{~mL}$ round bottom flask was added 3-methylbenzothiazole-2-thione $(2.0 \mathrm{~g}, 11$ mmol, 1.0 equiv.) and iodomethane (3.2 g, $23 \mathrm{mmol}, 2.0$ equiv.). Sealed with a rubber septa and heated at $50{ }^{\circ} \mathrm{C}$ for $4 \mathrm{~h}$ under an argon atmosphere. The reaction mixture became a white solid. After cooling to room temperature, the solid was suspended in $\mathrm{MeOH}(100 \mathrm{~mL})$ and $\mathrm{Et}_{2} \mathrm{O}(75 \mathrm{~mL})$ was added to ensure that the product had fully precipitated. The precipitate was collected via vacuum filtration and washed with $\mathrm{Et}_{2} \mathrm{O}$ (3 X $10 \mathrm{~mL})$. Dried under reduced pressure to give a white solid $(3.0 \mathrm{~g}, 84 \%)$. ${ }^{1} \mathbf{H} \mathbf{~ N M R}$ (400 MHz, DMSO) $\square 3.13$ (s, 3H, $\mathrm{SCH}_{3}$ ), 4.11 (s, 3H, $\mathrm{NCH}_{3}$ ), 7.70-7.77 (m, 1H, Ar), 7.82-7.88 (m, 1H, Ar), 8.20 (d, J = 8.42 Hz, 1H, Ar), 8.41 (d, J = 8.06 Hz, 1H, Ar); Exact mass calculated for $\left[\mathrm{C}_{9} \mathrm{H}_{10} \mathrm{NS}_{2}{ }^{+}\right]$requires $\mathrm{m} / \mathrm{z}$ 196.0249. Found 196.0251 (ESI+).

\section{Preparation of compound 2}

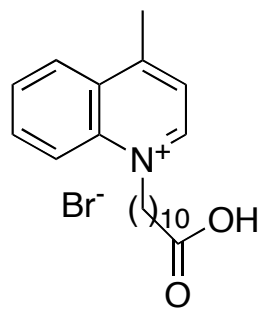

To a dry $100 \mathrm{~mL}$ round bottom flask was added 4-methylquinoline (2.0 g, $14 \mathrm{mmol}, 1.0$ equiv.) and 11-bromoundecanoic acid (4.1 g, $15 \mathrm{mmol}, 1.1$ equiv.). Sealed with a rubber septa and heated at $110^{\circ} \mathrm{C}$ for $3 \mathrm{~h}$ under an argon atmosphere. The resulting residue was dissolved in $\mathrm{MeOH}(20 \mathrm{~mL})$ and the product was precipitated upon addition of $\mathrm{Et}_{2} \mathrm{O}(40$ $\mathrm{mL})$. The precipitate was collected via vacuum filtration and washed with $\mathrm{Et}_{2} \mathrm{O}(3 \mathrm{X} 10$ $\mathrm{mL})$. Dried under reduced pressure to give a pink solid $(2.2 \mathrm{~g}, 38 \%)$. ${ }^{1} \mathbf{H}$ NMR (400

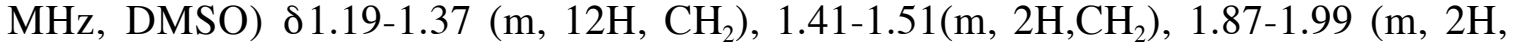
$\left.\mathrm{CH}_{2}\right), 2.17\left(\mathrm{t}, \mathrm{J}=7.33 \mathrm{~Hz}, 2 \mathrm{H}, \mathrm{CH}_{2}\right), 3.00\left(\mathrm{~s}, 3 \mathrm{H}, \mathrm{CH}_{3}\right), 5.00\left(\mathrm{t}, \mathrm{J}=7.51 \mathrm{~Hz}, 2 \mathrm{H}, \mathrm{NCH}_{2}\right)$, 8.02-8.10 (m, 2H, Ar), 8.22-8.30 (m, 1H, Ar), 8.51-8.63 (m, 2H, Ar), 9.41 (d, J = 6.23 $\mathrm{Hz}, 1 \mathrm{H}, \mathrm{Ar})$; Exact mass calculated for $\left[\mathrm{C}_{21} \mathrm{H}_{30} \mathrm{NO}_{2}{ }^{+}\right]$requires $m / z$ 328.2271. Found 328.2274 (ESI+). 


\section{Preparation of compound 3}

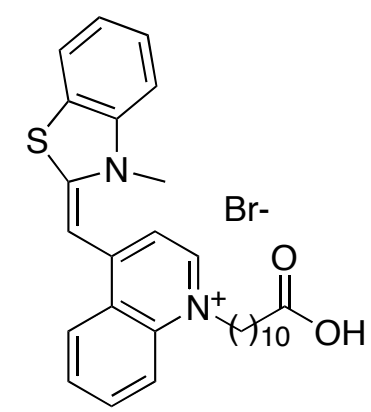

To a dry $100 \mathrm{~mL}$ round bottom flask was added compound 4 (1.0 g, $2.4 \mathrm{mmol}, 1.0$ equiv.) and compound 5 (0.79 g, $2.4 \mathrm{mmol}, 1.0$ equiv.) then dry EtOH $(24 \mathrm{~mL})$. To the resulting mixture was added dry $\mathrm{Et}_{3} \mathrm{~N}(0.74 \mathrm{~mL}, 5.2 \mathrm{mmol}, 2.2$ equiv.) resulting in an immediate deep red color. Heated at $55{ }^{\circ} \mathrm{C}$ for $2 \mathrm{~h}$ then at room temperature for $1 \mathrm{~h}$ under an argon atmosphere. The mixture was allowed to cool to room temperature then precipitated by addition of $\mathrm{Et}_{2} \mathrm{O}(50 \mathrm{~mL})$. The crude solid was suspended in $\mathrm{Me}_{2} \mathrm{CO}$ (70 $\mathrm{mL}) / \mathrm{Et}_{2} \mathrm{O}(100 \mathrm{~mL})$ for $1 \mathrm{~h}$ then collected via vacuum filtration and washed with $\mathrm{Et}_{2} \mathrm{O}(3$ $\mathrm{X} 10 \mathrm{~mL})$. Dried under reduced pressure to give a red solid $(0.77 \mathrm{~g}, 52 \%)$. ${ }^{1} \mathbf{H}$ NMR (400 MHz, DMSO) ?1.15-1.39 (m, 12H, $\left.\mathrm{CH}_{2}\right), 1.40-1.51\left(\mathrm{~m}, 2 \mathrm{H}, \mathrm{CH}_{2}\right), 1.78-1.91(\mathrm{~m}$, $\left.2 \mathrm{H}, \mathrm{CH}_{2}\right), 2.16\left(\mathrm{t}, \mathrm{J}=7.33 \mathrm{~Hz}, 2 \mathrm{H}, \mathrm{CH}_{2}\right), 4.03\left(\mathrm{~s}, 3 \mathrm{H}, \mathrm{CH}_{3}\right), 4.60(\mathrm{t}, \mathrm{J}=7.14 \mathrm{~Hz}, 2 \mathrm{H}$, $\left.\mathrm{NCH}_{2}\right), 6.94(\mathrm{~s}, 1 \mathrm{H}, \mathrm{CH}), 7.38(\mathrm{~d}, \mathrm{~J}=7.32 \mathrm{~Hz}, 1 \mathrm{H}, \mathrm{Ar}), 7.41-7.46(\mathrm{~m}, 1 \mathrm{H}, \mathrm{Ar}), 7.59-7.66$ (m, 1H, Ar), 7.73-7.83 (m, 2H, Ar), 7.97-8.03 (m, 1H, Ar), $8.06(\mathrm{~d}, \mathrm{~J}=7.69 \mathrm{~Hz}, 1 \mathrm{H}, \mathrm{Ar})$, $8.15(\mathrm{~d}, \mathrm{~J}=8.43 \mathrm{~Hz}, 1 \mathrm{H}, \mathrm{Ar}), 8.64(\mathrm{~d}, \mathrm{~J}=7.32 \mathrm{~Hz}, 1 \mathrm{H}, \mathrm{Ar}), 8.78(\mathrm{~d}, \mathrm{~J}=8.78 \mathrm{~Hz}, 1 \mathrm{H}, \mathrm{Ar})$ ${ }^{13}$ C NMR (125 MHz, DMSO) $\square 174.78,159.96,148.48,144.32,140.41,136.95,133.21$, $128.11,126.72,125.78,124.42,124.19,123.81,122.83,118.05,112.92,107.79,88.04$, 54.09, 34.51, 33.78, 28.71, 28.67, 28.65, 28.62, 28.39, 25.75, 24.79; Exact mass calculated for $\left[\mathrm{C}_{29} \mathrm{H}_{35} \mathrm{~N}_{2} \mathrm{O}_{2} \mathrm{~S}^{+}\right]$requires $m / z$ 475.2414. Found 475.2415 (ESI+); HPLC retention time $32.8 \mathrm{~min} ; \mathbf{m p}=164-166{ }^{\circ} \mathrm{C}$

\section{Preparation of compound 4}

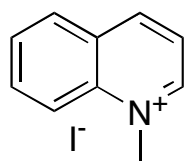

To a dry $100 \mathrm{~mL}$ round bottom flask was added quinoline ( $2.5 \mathrm{~g}, 19 \mathrm{mmol}, 1.0$ equiv.) and iodomethane $(8.8 \mathrm{~g}, 62 \mathrm{mmol}, 3.2$ equiv.). To the resulting mixture was added dry 1,4-dioxane $(40 \mathrm{~mL})$. Refluxed under an argon atmosphere for $1 \mathrm{~h}$. The reaction mixture was allowed to cool to room temperature and the solid was collected via vacuum filtration and washed with $\mathrm{Et}_{2} \mathrm{O}(3 \mathrm{X} 10 \mathrm{~mL})$ then petroleum ether $(3 \mathrm{X} 10 \mathrm{~mL})$. Dried under reduced pressure to give an orange solid $(4.4 \mathrm{~g}, 83 \%)$. ${ }^{1} \mathbf{H}$ NMR $(400 \mathrm{MHz}$, DMSO $\square 4.64\left(\mathrm{~s}, 3 \mathrm{H}, \mathrm{CH}_{3}\right), 8.05-8.10(\mathrm{~m}, 1 \mathrm{H}, \mathrm{Ar}), 8.15-8.20$ (m, $\left.1 \mathrm{H}, \mathrm{Ar}\right), 8.27-8.33(\mathrm{~m}$, $1 \mathrm{H}, \mathrm{Ar}), 8.47-8.54(\mathrm{~m}, 2 \mathrm{H}, \mathrm{Ar}), 9.29(\mathrm{~d}, \mathrm{~J}=8.42 \mathrm{~Hz}, 1 \mathrm{H}, \mathrm{Ar}), 9.51(\mathrm{~d}, \mathrm{~J}=5.86 \mathrm{~Hz}, 1 \mathrm{H}$, Ar); Exact mass calculated for $\left[\mathrm{C}_{10} \mathrm{H}_{10} \mathrm{~N}^{+}\right]$requires $m / z$, 144.0808. Found 144.0810 (ESI+). 


\section{Preparation of compound 5}

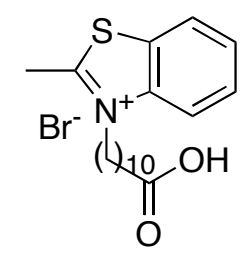

To a dry $100 \mathrm{~mL}$ round bottom flask was added 2-methylbenzothiazole $(7.0 \mathrm{~g}, 47 \mathrm{mmol}$, 1.0 equiv.) and 11-bromoundecanoic acid ( $14 \mathrm{~g}, 52 \mathrm{mmol}, 1.1$ equiv.). Sealed with a rubber septa and heated at $110{ }^{\circ} \mathrm{C}$ for $5 \mathrm{~h}$ under an argon atmosphere. The resulting residue was dissolved in $\mathrm{MeOH}(20 \mathrm{~mL})$ and the product was precipitated upon addition of $\mathrm{Et}_{2} \mathrm{O}(40 \mathrm{~mL})$. The precipitate was collected via vacuum filtration and washed with $\mathrm{Et}_{2} \mathrm{O}(3 \times 10 \mathrm{~mL})$. Dried under reduced pressure to give a pink solid $(2.8 \mathrm{~g}, 15 \%)$. ${ }^{1} \mathbf{H}$ NMR (400 MHz, DMSO) $\square 1.19-1.37\left(\mathrm{~m}, 12 \mathrm{H}, \mathrm{CH}_{2}\right), 1.38-1.52\left(\mathrm{~m}, 2 \mathrm{H}, \mathrm{CH}_{2}\right), 1.78-1.89$ $\left(\mathrm{m}, 2 \mathrm{H}, \mathrm{CH}_{2}\right), 2.18\left(\mathrm{t}, \mathrm{J}=7.32 \mathrm{~Hz}, 2 \mathrm{H}, \mathrm{CH}_{2}\right), 3.21\left(\mathrm{~s}, 3 \mathrm{H}, \mathrm{CH}_{3}\right), 4.70(\mathrm{t}, \mathrm{J}=7.69 \mathrm{~Hz}, 2 \mathrm{H}$, $\left.\mathrm{NCH}_{2}\right), 7.76-7.84(\mathrm{~m}, 1 \mathrm{H}, \mathrm{Ar}), 7.85-7.93(\mathrm{~m}, 1 \mathrm{H}, \mathrm{Ar}), 8.33(\mathrm{~d}, \mathrm{~J}=8.42 \mathrm{~Hz}, 1 \mathrm{H}, \mathrm{Ar}), 8.44$ $(\mathrm{d}, \mathrm{J}=8.06 \mathrm{~Hz}, 1 \mathrm{H}, \mathrm{Ar})$; Exact mass calculated for $\left[\mathrm{C}_{19} \mathrm{H}_{28} \mathrm{NO}_{2} \mathrm{~S}^{+}\right]$requires $\mathrm{m} / \mathrm{z}$ 334.1835. Found 334.1841 (ESI+).

\section{Preparation of compound 6}

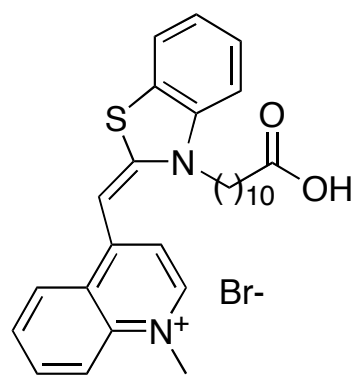

To a dry $100 \mathrm{~mL}$ round bottom flask was added compound $1(1.9 \mathrm{~g}, 7.1 \mathrm{mmol}, 1.0$ equiv.) and compound $2(2.9 \mathrm{~g}, 7.1 \mathrm{mmol}, 1.0$ equiv.) then dry DCM $(30 \mathrm{~mL})$. To the resulting mixture was added dry $\mathrm{Et}_{3} \mathrm{~N}(2.9 \mathrm{~mL}, 21 \mathrm{mmol}, 3.0$ equiv.) resulting in an immediate deep red color. Stirred at room temperature under an argon atmosphere for 48 $\mathrm{h}$ then concentrated in vacuo to give a deep red residue. The residue was dissolved in $\mathrm{EtOH}(20 \mathrm{~mL})$ then $\mathrm{Et}_{2} \mathrm{O}(5 \mathrm{~mL})$ was added. Stored in the refrigerator for $15 \mathrm{~h}$. The crude product was collected via vacuum filtration and washed with $\mathrm{Et}_{2} \mathrm{O}(3 \mathrm{X} 10 \mathrm{~mL})$. Dissolved the red solid in $\mathrm{MeOH}(20 \mathrm{~mL})$ then $\mathrm{H}_{2} \mathrm{O}(5 \mathrm{~mL})$ was added. Stored in the refrigerator for $24 \mathrm{~h}$. The precipitate was collected via vacuum filtration and washed with $\mathrm{H}_{2} \mathrm{O}(3 \mathrm{X} 10 \mathrm{~mL})$. The solid was suspended in $\mathrm{Me}_{2} \mathrm{CO}(10 \mathrm{~mL})$ for $1 \mathrm{~h}$ then collected via vacuum filtration and washed with $\mathrm{Me}_{2} \mathrm{CO}(3 \mathrm{X} 10 \mathrm{~mL})$. Dried under reduced pressure to give a red solid $(0.46 \mathrm{~g}, 12 \%)$. ${ }^{1} \mathbf{H}$ NMR $\left(500 \mathrm{MHz}, \mathrm{CD}_{3} \mathrm{OD}\right)$ 01.19-1.46 (m, 12H, $\left.\mathrm{CH}_{2}\right), 1.47-1.55\left(\mathrm{~m}, 2 \mathrm{H}, \mathrm{CH}_{2}\right), 1.87-1.96\left(\mathrm{~m}, 2 \mathrm{H}, \mathrm{CH}_{2}\right), 2.15(\mathrm{t}, \mathrm{J}=$ $\left.7.52 \mathrm{~Hz}, 2 \mathrm{H}, \mathrm{CH}_{2}\right), 4.16\left(\mathrm{~s}, 3 \mathrm{H}, \mathrm{CH}_{3}\right), 4.53\left(\mathrm{t}, \mathrm{J}=7.33 \mathrm{~Hz}, 2 \mathrm{H}, \mathrm{NCH}_{2}\right), 6.88(\mathrm{~s}, 1 \mathrm{H}, \mathrm{CH})$, 7.35-7.48 (m, 2H, Ar), 7.55-7.64 (m, 2H, Ar), 7.74-7.80 (m, 1H, Ar), $7.87(\mathrm{~d}, \mathrm{~J}=7.70$ $\mathrm{Hz}, 1 \mathrm{H}, \mathrm{Ar}), 7.95-8.03(\mathrm{~m}, 2 \mathrm{H}, \mathrm{Ar}), 8.36(\mathrm{~d}, \mathrm{~J}=6.96 \mathrm{~Hz}, 1 \mathrm{H}, \mathrm{Ar}), 8.55(\mathrm{~d}, \mathrm{~J}=8.43 \mathrm{~Hz}$, $1 \mathrm{H}, \mathrm{Ar}) ;{ }^{13} \mathrm{C}$ NMR $\left(125 \mathrm{MHz}, \mathrm{CD}_{3} \mathrm{OD}\right) \square$ 179.98, 161.57, 150.99, 145.79, 141.63, 139.97, 134.68, 129.66, 128.52, 126.24, 126.08, 125.96, 125.89, 123.92, 119.16, 114.06, 
109.73, 89.08, 47.47, 43.24, 36.90, 30.54, 30.52, 30.47, 30.38, 28.37, 27.73, 26.87; Exact mass calculated for $\left[\mathrm{C}_{29} \mathrm{H}_{35} \mathrm{~N}_{2} \mathrm{O}_{2} \mathrm{~S}^{+}\right]$requires $\mathrm{m} / \mathrm{z}$ 475.2414. Found 475.2416 (ESI+); HPLC retention time $38.1 \mathrm{~min} ; \mathbf{m p}=144-146^{\circ} \mathrm{C}$

\section{General protocol for the synthesis of TO-peptide conjugates}

TO-peptide conjugates were synthesized on solid support using commercially available Wang-Fmoc-Lys(Boc) $(0.7 \mathrm{mmol} / \mathrm{g}$, Advanced ChemTech). Couplings were performed using 4 equivalents of Fmoc protected amino acid, 4 equivalents of HBTU and 8 equivalents of diisopropylethylamine in DMF for 3 hours. Deprotection of the Fmoc group was achieved using 20\% piperidine in DMF for 30 minutes (after coupling of the first amino acid to the resin, Fmoc deprotection was achieved using 50\% piperidine in DMF for 5 minutes in order to minimize diketopiperazine formation). The TO-peptide conjugates (TO) were simultaneously deprotected and cleaved from the resin with a solution of 9:1:1:1 trifluoracetic acid: triethylsilane: triisopropylethylamine: $\mathrm{H}_{2} \mathrm{O}$ for 30 minutes. The solution was concentrated under reduced pressure in the presence of toluene in order to remove any residual TFA. TO-peptide conjugates were dissolved in a minimal amount of methanol, which was then removed under vacuum to yield a red solid.

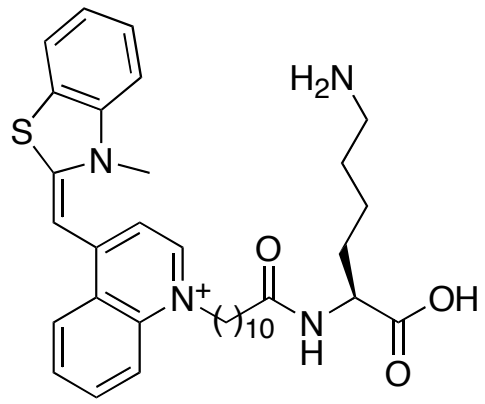

$\mathbf{T O}_{\mathrm{Q}}-\mathrm{K}(\mathbf{3 a})$

Exact mass calculated for $\left[\mathrm{C}_{35} \mathrm{H}_{47} \mathrm{~N}_{4} \mathrm{O}_{3} \mathrm{~S}^{+}\right]$requires $\mathrm{m} / \mathrm{z}$ 603.3363. Found 603.3366 (ESI+); HPLC retention time: $31.1 \mathrm{~min}$

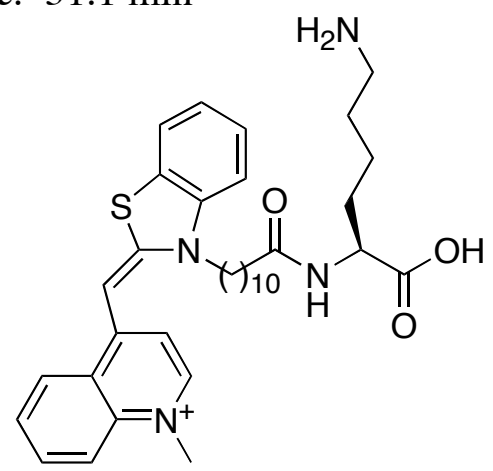

$\mathrm{TO}_{\mathrm{z}}-\mathrm{K}(\mathbf{6 a})$

Exact mass calculated for $\left[\mathrm{C}_{35} \mathrm{H}_{47} \mathrm{~N}_{4} \mathrm{O}_{3} \mathrm{~S}^{+}\right]$requires $\mathrm{m} / \mathrm{z}$ 603.3363. Found 603.3363 (ESI+); HPLC retention time: $29.2 \mathrm{~min}$ 


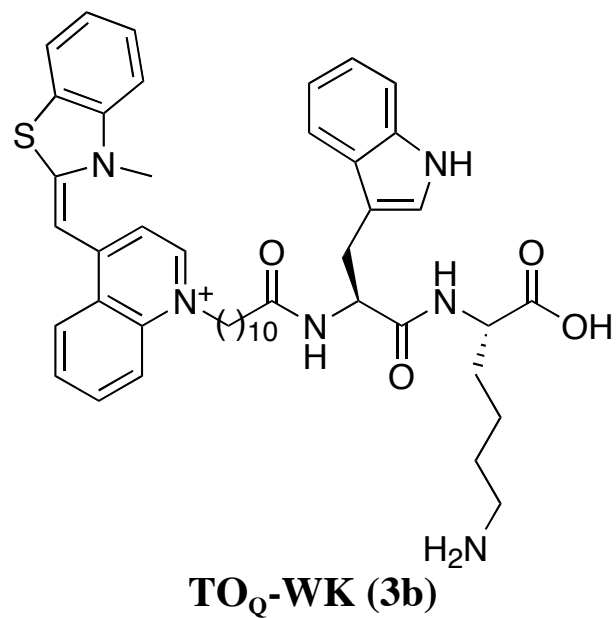

Exact mass calculated for $\left[\mathrm{C}_{46} \mathrm{H}_{57} \mathrm{~N}_{6} \mathrm{O}_{4} \mathrm{~S}^{+}\right]$requires $\mathrm{m} / \mathrm{z}$ 789.4157. Found 789.4178 (ESI+); HPLC retention time: $39.2 \mathrm{~min}$

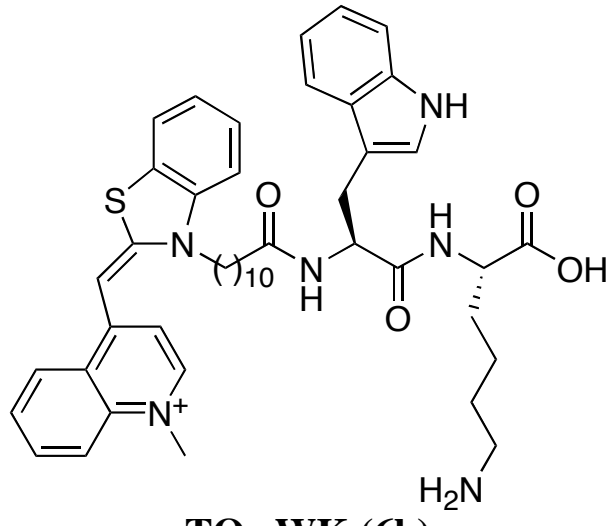

$\mathrm{TO}_{\mathrm{z}}-\mathrm{WK}(\mathbf{6 b})$

Exact mass calculated for $\left[\mathrm{C}_{46} \mathrm{H}_{57} \mathrm{~N}_{6} \mathrm{O}_{4} \mathrm{~S}^{+}\right]$requires $\mathrm{m} / \mathrm{z}$ 789.4157. Found 789.4156 (ESI+); HPLC retention time: $35.6 \mathrm{~min}$<smiles>CN1/C(=C\c2cc[n+](CC(=O)OI)c3ccccc23)Sc2ccccc21</smiles><smiles>CCCCC[C@H](NC(=O)O)C(=O)N[C@@H](C)C(=O)N[C@@H](CCC(N)=O)C(=O)N[C@@H](CO)C(N)=O</smiles><smiles>NCC(Cc1c[nH]c2ccccc12)C(=O)NCC(=O)O</smiles>

TO<smiles>CN[C@@H](CCCCN)C(=O)N[C@@H](CO)C(=O)NC(C)C(=O)N[C@@H](CCCCN)C(=O)O</smiles>

Exact mass calculated for $\left[\mathrm{C}_{77} \mathrm{H}_{113} \mathrm{~N}_{17} \mathrm{O}_{15} \mathrm{~S}^{2+}\right]$ requires $\mathrm{m} / \mathrm{z} 773.9156$. Found 773.9142 (ESI+); HPLC retention time: $31.6 \mathrm{~min}$ 
<smiles>CC(NC(=O)[C@H](CO)NC(=O)[C@H](CCCCN)NC(=O)CNC(=O)[C@H](Cc1c[nH]c2ccccc12)NC(=O)[C@H](CO)NC(=O)[C@H](CCC(N)=O)NC(=O)[C@H](C)NC(=O)[C@H](CCCCN)NC(=O)NN1/C(=C\c2cc[n+](C)c3ccccc23)Sc2ccccc21)C(=O)N[C@H](C)C(=O)O</smiles>

\section{TO $_{\mathrm{z}^{-}}$KAQSWGKSAK (6c)}

Exact mass calculated for $\left[\mathrm{C}_{77} \mathrm{H}_{113} \mathrm{~N}_{17} \mathrm{O}_{15} \mathrm{~S}^{2+}\right]$ requires $\mathrm{m} / \mathrm{z} 773.9156$. Found 773.9153 (ESI+); HPLC retention time: $36.0 \mathrm{~min}$<smiles>CC(C)[C@H](NC(=O)[C@H](CCC(N)=O)NC(=O)[C@H](C)NC(=O)CNC(=O)[Y6][n+]1ccc(/C=C2/Sc3ccccc3N2C)c2ccccc21)C(=O)N[C@@H](Cc1c[nH]c2ccccc12)C(=O)NCC(=O)N[C@@H](Cc1ccccc1)C(=O)N[C@@H](CO)C(=O)N[C@@H](C)C(=O)N[C@@H](CCCCN)C(=O)O</smiles>

\section{TO $\mathbf{Q}^{-G A Q V W G F S A K ~(3 d) ~}$}

Exact mass calculated for $\left[\mathrm{C}_{78} \mathrm{H}_{105} \mathrm{~N}_{15} \mathrm{O}_{14} \mathrm{~S}^{2+}\right]$ requires $\mathrm{m} / \mathrm{z} 753.8838$. Found 753.8835 (ESI+); HPLC retention time: $27.4 \mathrm{~min}$

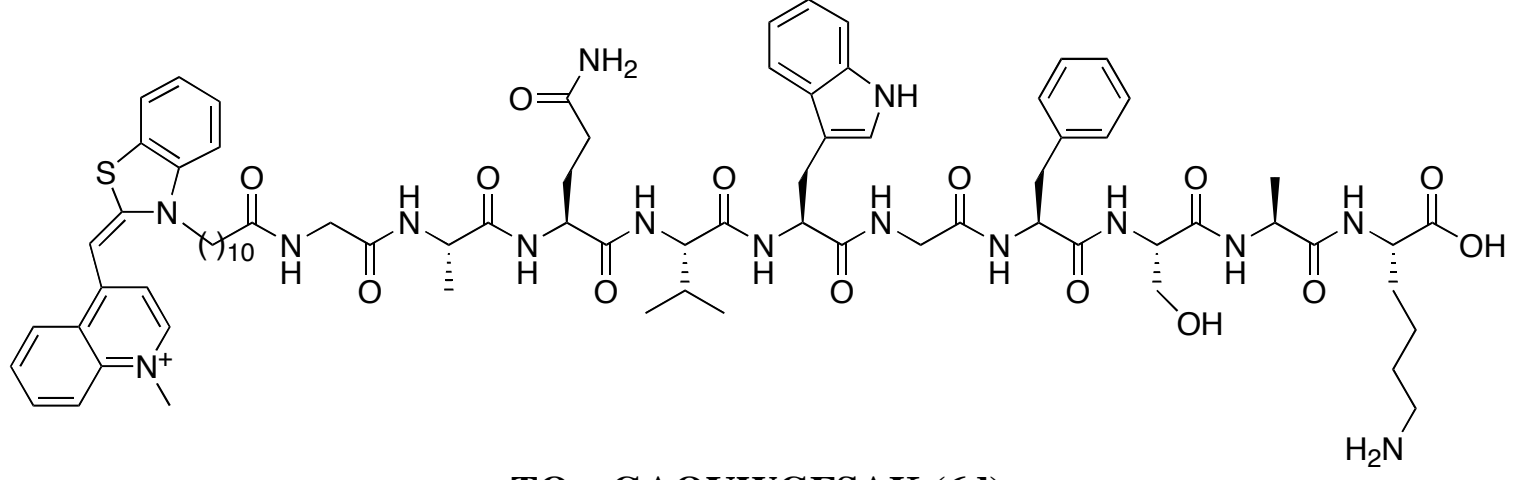

TO $\mathrm{Z}^{-}$GAQVWGFSAK (6d)

Exact mass calculated for $\left[\mathrm{C}_{78} \mathrm{H}_{105} \mathrm{~N}_{15} \mathrm{O}_{14} \mathrm{~S}^{2+}\right]$ requires $\mathrm{m} / \mathrm{z} 753.8838$. Found 753.8839 (ESI+); HPLC retention time: $28.6 \mathrm{~min}$ 


\section{References}

[1] Weber, G.; Teale, F. W. J. Trans. Faraday Soc. 1957, 53, 646.

[2] http://www.graphpad.com/

[3] Netzel, T. L.; Nafisi, K.; Zhao, M.; Lenhard, J. R.; Johnson, I. J. Phys. Chem. 1995, 99, 17936.

[4] Nygren, T. L.; Svanvik, N.; Kubista, M. Biopolymers, 1998, 46, 39.

[5] Boger, D. L.; Tse, W. C.; Bioorg. Med. Chem. 2001, 9, 2511.

[6] Boger, D. L.; Fink, B. E.; Brunette, S. R.; Tse, W. C.; Hedrick, M. P. J. Am. Chem. Soc. 2001, 123, 5878.

[7] (a) Svanvik, N.; Westman, G.; Wang, D.; Kubista, M. Anal. Biochem. 2000, 281, 26. (b) Benson, S. C.; Singh, P.; Glazer, A. N. Nucleic Acids Res. 1993, 21, 5727. (c) Privat, E.; Asseline, U. Bioconj. Chem. 2001, 12, 757. 